\title{
Calculations of Branching Ratios for Radiative-Capture, One-Proton, and Two-Neutron Channels in the Fusion Reaction ${ }^{209} \mathbf{B i}+{ }^{70} \mathbf{Z n}$
}

\author{
Takatoshi Ichikawa and Akira Iwamoto ${ }^{1}$ \\ Yukawa Institute for Theoretical Physics, Kyoto University, Kyoto 606-8502, Japan \\ ${ }^{1}$ Japan Atomic Energy Agency, Tokai-mura, Naka-gun, Ibaraki 319-1195, Japan
}

\begin{abstract}
We discuss the possibility of the non-one-neutron emission channels in the cold fusion reaction ${ }^{70} \mathrm{Zn}$ $+{ }^{209} \mathrm{Bi}$ to produce the element $Z=113$. For this purpose, we calculate the evaporation-residue cross sections of one-proton, radiative-capture, and two-neutron emissions relative to the one-neutron emission in the reaction ${ }^{70} \mathrm{Zn}+{ }^{209} \mathrm{Bi}$. To estimate the upper bounds of those quantities, we vary model parameters in the calculations, such as the level-density parameter and the height of the fission barrier. We conclude that the highest possibility is for the $2 \mathrm{n}$ reaction channel, and its upper bounds are $2.4 \%$ and at most less than $7.9 \%$ with unrealistic parameter values, under the actual experimental conditions of [J. Phys. Soc. Jpn. 73 (2004) 2593].
\end{abstract}

KEYWORDS: nuclear reaction, superheavy element, cold fusion reaction, deexcitation, decay width

\section{Introduction}

Heavy-ion fusion reactions provide a powerful tool for synthesizing superheavy elements (SHE). ${ }^{1,2)}$ To verify that a new element has been created in such reactions, it is necessary to identify which evaporation residue is formed when the excited fusion product, in competition with nuclear fission, is de-excited by particle or gamma-ray emissions to a certain stable state. In this paper, we focus on the experiment performed by Morita et al. to produce the element $113 .^{3,4)}$ The reaction channel of this experiment for the observed two alpha chains was assigned as the one-neutron (1n) evaporation channel. We investigate here the sensitivity of this assignment to different model assumptions.

The synthesized elements can be identified by measuring the alpha-decay chains to known nuclei. However, this is not always straightforward in the case of odd-odd evaporation residues, such as those in the experiments cited in refs. 3 and 4. In such alpha-decay chains, one can expect not only ground-state-to-ground-state alpha decay but also the involvement of excited states. Therefore, it is difficult to compare directly the observed alpha-decay energy and lifetime with the measured values of known products in the decay chain. Uncertainties coming from experimental conditions may also lead to difficulties in identifications. Morita et al. have recently performed an experiment on the formation of the nucleus ${ }^{266} \mathrm{Bh}^{5)}$ which is the grand-grand daughter of the nucleus ${ }^{278} 113$, and observed its decay modes. From the comparison of the alpha-decay chain of ${ }^{266} \mathrm{Bh}$ with that of ${ }^{278} 113$, they further confirmed the $1 \mathrm{n}$ reaction channel assignment.

In addition to these experimental studies, it is desirable to calculate the confidence level with which other reaction channels can be excluded from the reaction mechanism consideration. We therefore calculate the probabilities of the competing reaction channels relative to the 1 channel in standard theoretical models. Although the calculation is performed only for this special example, this approach can be applied to any other experiments for the SHE production based on cold fusion reactions, offering a simple and practical estimator.

In practice, for the synthesis of the nucleus ${ }^{278} 113$ using the ${ }^{209} \mathrm{Bi}\left({ }^{70} \mathrm{Zn}, \mathrm{n}\right)$ reaction, ${ }^{3)}$ Morita et al. excluded the follow- ing possibilities: (i) radiative capture process (zero-neutron evaporation channel) leading to the nucleus ${ }^{279} 113$, (ii) twoneutron evaporation channel leading to the nucleus ${ }^{277} 113$, and (iii) one-proton evaporation channel leading to the $n u$ cleus ${ }^{278} 112$. We will quantitatively estimate the probability of these decay channels by calculating the evaporationresidue (ER) cross section.

For such estimations, it is difficult to calculate the non1n reaction using the Monte-Carlo simulation for the deexcitation process because of its extremely low survival probability. In this respect, we employ the "fusion-by-diffusion" (FBD) model, 6,7) which consists of simple analytic expressions and is well optimized for various cold fusion reactions. We extend it to the non- $1 n$ reaction. One difficulty in the estimation is that the de-excitation process is very sensitive to the level-density parameter and the height of the fission barrier. To reduce these uncertainties, we estimate worst-case scenarios for relative probabilities by varying substantially the leveldensity parameter and the height of the fission barrier. We demonstrate below that the assignment given to the experiment cited in ref. 3 is highly probable.

This paper is organized as follows: In $\S 2$, we describe details of models and parameters for numerical calculations. In $\S 3$, we show the calculated results and discuss the probability of the radiative capture, two-neutron evaporation, and oneproton evaporation channels. Finally, we summarize in $\S 4$ the confidence level with which the evaporation residues of the experiments cited in refs. 3 and 4 can be assigned to the nucleus ${ }^{278} 113$.

\section{Model and Input Parameters}

In this study, all calculations are based on the (FBD) model proposed by Świątecki et al. ${ }^{6,7)}$ and its straightforward extensions, because their model and parameters have been well optimized for various cold-fusion reactions and reproduced well the experimental data. ${ }^{8-14)}$ The evaporation residue (ER) cross section $\sigma_{\mathrm{ER}}$ is given by

$$
\sigma_{\mathrm{ER}}=\sigma_{\mathrm{cap}} \cdot P_{\mathrm{diff}} \cdot W_{\mathrm{suv}}
$$


Table I. Fission barrier heights $B_{\mathrm{f}}$ obtained from ref. 23 and oneneutron and one-proton separation energies $S_{1 \mathrm{n}}$ and $S_{1 \mathrm{p}}$ estimated with FRDM95. ${ }^{19)}$

\begin{tabular}{cccc}
\hline Nuclide & $B_{\mathrm{f}}(\mathrm{MeV})$ & $S_{1 \mathrm{n}}(\mathrm{MeV})$ & $S_{1 \mathrm{p}}(\mathrm{MeV})$ \\
\hline${ }^{279} 113$ & 6.12 & 7.37 & 0.48 \\
${ }^{278} 113$ & 6.06 & 6.32 & \\
${ }^{277} 113$ & 6.31 & 7.59 & \\
${ }^{278} 112$ & 5.99 & 7.14 & \\
\hline
\end{tabular}

where $\sigma_{\text {cap }}$ is the capture cross section, $P_{\text {diff }}$ is the probability that two touching nuclei can reach the compound state, which is descried as "diffusion" driven by thermal fluctuations of collective degrees of freedoms, and $W_{\text {suv }}$ is the survival probability of the excited compound nucleus.

One characteristic feature of this model is that the neutron decay width is calculated with the "transition state" method, ${ }^{7,15-17)}$ rather than the traditional "detailedbalance" method. ${ }^{18)}$ They showed that the neutron evaporation width evaluated by the detailed-balance method with the temperature-dependent shell correction energy is incorrectly suppressed near the fission threshold. ${ }^{15)}$ We apply the transition state method to the proton evaporation channel as well.

For the nuclear mass, the finite-range droplet model $\left(\right.$ FRDM95) ${ }^{19)}$ and the Hartree-Fock-Bogoliubov model $(\mathrm{HFB} 02)^{20)}$ have been recommended in the reference input parameter library (RIPL-2). ${ }^{21)}$ In the FBD model, the Thomas-Fermi model developed by Myers and Świạtecki (MS96) $^{22)}$ was used. Among those models, we chose FRDM95 as the main model in this work, because not only the mass excess but also the shell correction energies and the fission barrier heights are consistently provided. Although we use the parameters optimized for FRDM95 in all calculations, we investigate the worst-case scenario at the different excitation energies estimated with the three mass models, which also causes uncertainty in this study, as will be discussed in $\S 2.1$.

We calculate the level-density parameters $a$ with eq. (A10) in ref. 7. The uncertainty of the level density with the shell correction energy of FRDM95 was extensively investigated in a previous study cited in ref. 21. In RIPL-2, it was shown that the expected uncertainty for an unknown level-density parameter is about $6.5 \%$. We thus vary the asymptotic level-density parameter by up to $10 \%$ (see also Fig. 6.4 in ref. 21).

We use the height of the fission barrier tabulated in ref. 23 and the one-neutron and one-proton separation energies calculated with FRDM95. The values used in the calculation are tabulated in Table I. We also analyze the neutron separation energies calculated with the other two models and observe a small difference. For the uncertainty of the fission barrier height, the root-mean square deviation of the calculated results from the experimental data is shown to be $0.99 \mathrm{MeV}$ in refs. 23 and 24; thus, we increase or decrease the height of the fission barrier by $\pm 1 \mathrm{MeV}$. We also simultaneously increase or decrease the shell correction energy, because the fission barrier in the superheavy-mass region is mainly due to the shell correction energy at the ground state.

For calculations of $P_{\mathrm{diff}}$, we estimate the temperature at the saddle point with the fission barrier heights given in Table I. All the other calculations are the same as those in ref. 7. Because the fission barrier heights differ from the original cal-

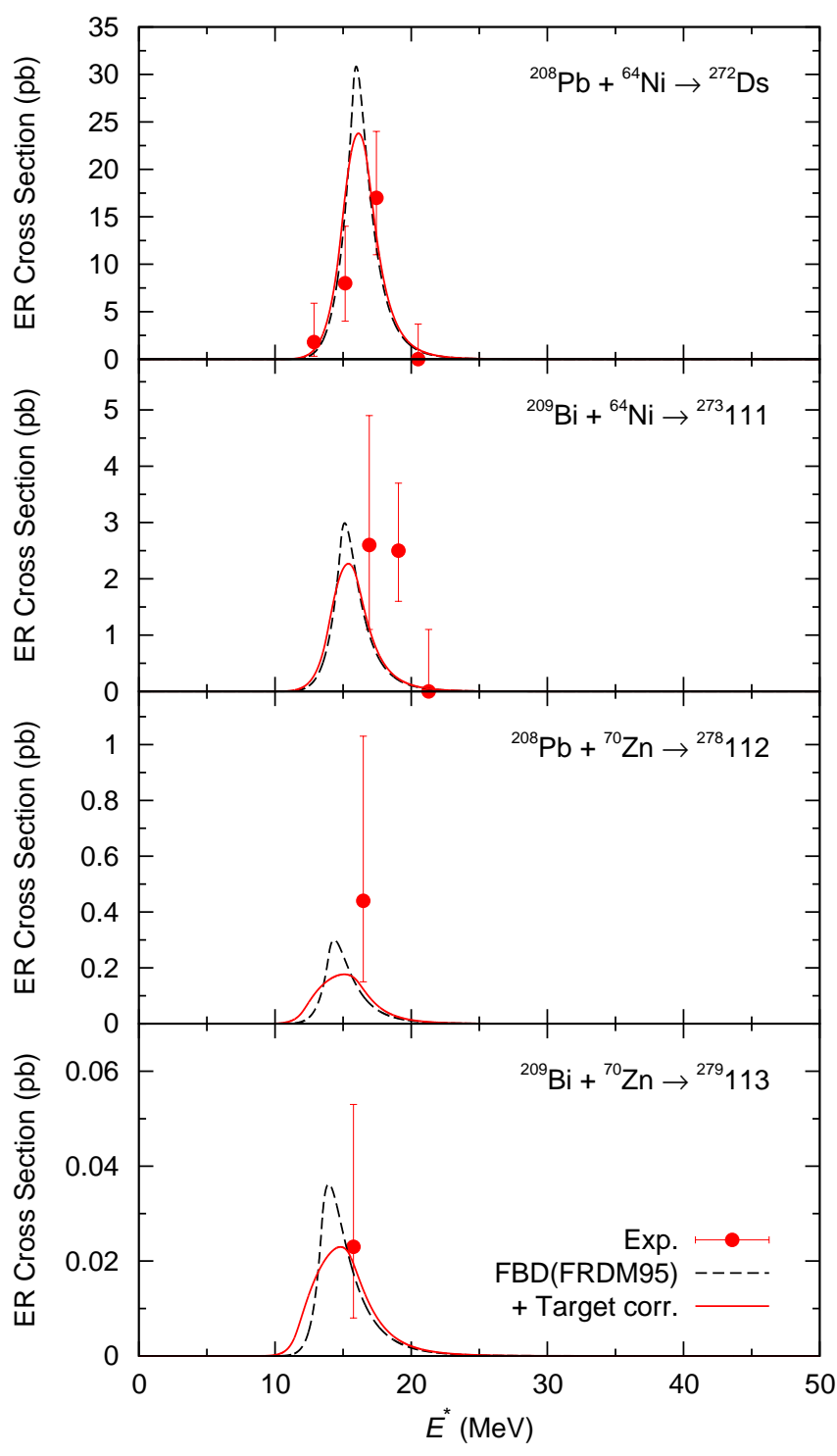

Fig. 1. (Color online) Evaporation residue cross sections for the 1n reaction channel in various cold fusion reactions calculated with the FBD model. The solid and dashed lines respectively indicate the calculated results with and without the correction due to the target thickness. The solid circle indicates the experimental data obtained from refs. 25-28.

culations in ref. 7, we need to fine-tune the neck parameter $s$, which is only one adjustable parameter in the FBD model. We choose $s=2.1 \mathrm{fm}$ in order to fit the experimental data for the cold fusion reactions performed by RIKEN and obtain an overall fit for the experimental data, as shown in Fig. 1. In the figure, the solid and dashed lines respectively indicate the calculated results of the ER cross section with and without the target correction due to the target thickness under actual experimental conditions (as will be discussed in \$2.7). For comparison with the non- $1 \mathrm{n}$ reaction channels, we fix all parameters for the $1 \mathrm{n}$ reaction channel.

\subsection{Range of excitation energies}

One large uncertainty in estimations of the excitation energy of a formed compound nucleus is due to large differences between various mass models. For comparison, we calculate the excitation energies of the compound nucleus ${ }^{279} 113$ in the ${ }^{209} \mathrm{Bi}+{ }^{70} \mathrm{Zn}$ reaction with FRDM95, MS96, and HFB02. For 
the mass excesses of ${ }^{209} \mathrm{Bi}$ and ${ }^{70} \mathrm{Zn}$, we use the experimental masses obtained from Audi2003. ${ }^{29)}$ Since the experimental incident beam energy is $261.4 \mathrm{MeV}$, the resultant excitation energies are 15.76, 14.11 and 13.61 MeV for FRDM95, MS96, and HFB02, respectively. Note that in the experiment, the uncertainty of the incident beam energies is $\pm 2 \mathrm{MeV}$ due to the target thickness. ${ }^{3)}$ We thus vary the excitation energies in the range from 11.61 to $17.76 \mathrm{MeV}$ in this study.

\subsection{Proton decay width}

We extend the transition state method to the proton decay. The particle decay width is then given as

$$
\Gamma_{p}=\frac{D}{2 \pi} \int_{0}^{U^{*}-B_{\mathrm{p}}-V_{c}} \rho_{F}^{(p)}\left(U^{*}-B_{\mathrm{p}}-V_{c}-\epsilon\right) d \epsilon,
$$

where $B_{\mathrm{p}}$ is the binding energy of an evaporated proton, $V_{c}$ is the height of the Coulomb barrier, and $\rho_{F}$ is the level density for the final state. The symbol $U^{*}$ is the effective excitation energy, which is defined by $U^{*}=E^{*}-\Delta$, where $E^{*}$ is the excitation energy and $\Delta$ is the pairing energy normalized to zero for odd-odd nuclei as given explicitly in APPENDIX B of ref. 7. The symbol $D$ is $1 / \rho_{I}$, where $\rho_{I}$ is the level density for the initial state.

In the calculation, the height of the Coulomb barrier, $V_{c}$, has a large uncertainty, due to the difficulty in its direct measurement. In the standard statical model, $V_{c}$ is estimated from the fusion barrier of its inverse reaction. The empirical fusion barrier $B_{\text {fus }}$ is often taken as ${ }^{30}$ )

$$
B_{\text {fus }}=\frac{1.44(Z-1)}{1.18(A-1)^{1 / 3}+3.928} .
$$

However, Moretto pointed out that, in a heavy-mass system, the height of the Coulomb barrier for a proton emission is lower than that of the inverse reaction due to the polarization of many protons, ${ }^{31)}$ and Parker et al. proposed a new empirical formula extracted from the experimental systematics, ${ }^{32}$ ) given by $B_{\text {fus }}=0.106 Z-0.90$. For the nucleus ${ }^{279} 113$, the heights of the Coulomb barriers are 13.87 and $11.08 \mathrm{MeV}$ for the standard and new empirical formulas, respectively. In this paper, we use $V_{c}=11.08 \mathrm{MeV}$ to discuss the worst-case scenario.

\section{$2.3 \gamma$-Ray decay width}

For the gamma-decay width, we obtain equations and their parameters from ref. 33; however, for simplicity, we ignore the angular-momentum dependence and consider only the E1 transition. The gamma-decay width is thus given by

$$
\Gamma_{\gamma}=\hbar \int_{0}^{U^{*}} w_{\gamma}\left(U^{*}, \epsilon_{\gamma}\right) d \epsilon_{\gamma},
$$

where the transition probability $w_{\gamma}$ for the E1 gamma-ray emission is given by

$$
w_{\gamma}\left(U^{*}, \epsilon_{\gamma}\right)=\frac{1}{\hbar} \frac{\rho_{F}\left(U^{*}-\epsilon_{\gamma}\right)}{\rho_{I}\left(U^{*}\right)} \epsilon_{\gamma}^{3} f_{\mathrm{E} 1}\left(\epsilon_{\gamma}\right) .
$$

The function $f_{\mathrm{E} 1}$ is the gamma-strength function. Following ref. $33, f_{\mathrm{E} 1}$ was chosen as the following Lorentzian function:

$$
f_{\mathrm{E} 1}=3.31 \times 10^{-6} \mathrm{MeV}^{-1} \frac{N Z}{A} \frac{\epsilon_{\gamma} \Gamma}{\left(E_{0}^{2}-\epsilon_{\gamma}^{2}\right)^{2}+\epsilon_{\gamma}^{2} \Gamma^{2}},
$$

where we consider the values of the width $\Gamma$ and the resonance energy $E_{0}$ calculated using the droplet model: ${ }^{34)} \Gamma=5 \mathrm{MeV}$ and $E_{0}$ is given by

$$
E_{0}=167.23 A^{-1 / 3}\left(1.959+14.074 A^{-1 / 3}\right)^{-1 / 2} \mathrm{MeV}
$$

\subsection{Survival probability for one-proton emission}

We calculate the survival probability for the $1 \mathrm{n}$ reaction with the FBD model and extend it to the $1 \mathrm{p}$ and $2 \mathrm{n}$ reactions. In ref. 7 , the survival probability for the $1 \mathrm{n}$ reaction, $W_{\text {suv }}^{(1 \mathrm{n})}$, is expressed as the product of the probability for the one-neutron evaporation and the survival probability against the secondchance fission or the second-chance neutron emission, $W_{<}^{(2 n d)}$, such that we extend it to the survival probability for the $1 p$ reaction, $W_{\text {suv }}^{(1 \mathrm{p})}$, given by

$$
W_{\text {suv }}^{(1 \mathrm{p})}\left(E^{*}\right)=\frac{\Gamma_{\mathrm{p}}\left(E^{*}\right)}{\Gamma_{\mathrm{Tot}}\left(E^{*}\right)} W_{<}^{(2 \mathrm{nd})}\left(E^{*}\right),
$$

where $\Gamma_{\text {Tot }}$ is the total decay width defined by $\Gamma_{\text {Tot }}=\Gamma_{\mathrm{n}}+\Gamma_{\mathrm{f}}+$ $\Gamma_{\mathrm{p}}+\Gamma_{\gamma}$. By assuming a standard proton evaporation spectrum proportional to $k \exp (-k / T)$, where $k$ is the neutron kinetic energy and $T$ is the temperature of the transition state for proton emission, $W_{<}^{(2 n d)}$ is given by

$$
W_{<}^{(2 \mathrm{nd})}= \begin{cases}\left(1+\frac{K}{T}\right) \exp \left(-\frac{K}{T}\right) & K \geqq 0, \\ 1 & K<0,\end{cases}
$$

where $K=U^{*}-B_{\mathrm{p}}-V_{C}-E_{\mathrm{th}}$ and $E_{\mathrm{th}}$ is the threshold energy for the second-chance fission or second-chance neutron emission whichever is lower.

\subsection{Survival probability for two-neutron emission}

For the $2 \mathrm{n}$ reaction, we assume that the survival probability is given by the product of $\Gamma_{\mathrm{n}}^{(1 \mathrm{n})} / \Gamma_{\mathrm{Tot}}^{(1 \mathrm{n})}$ for the first neutron evaporation, $\Gamma_{\mathrm{n}}^{(2 \mathrm{n})} / \Gamma_{\mathrm{Tot}}^{(2 n)}$ for the second neutron evaporation, and the survival probability against the third-chance fission or neutron emission. Since the second neutron evaporation width depends on the kinetic energy of the first emitted neutron, we approximate the probability of the second neutron emission using the ensemble average in the first neutron emission spectrum. $W_{\text {suv }}^{(2 n)}$ is thus given as

$$
\begin{gathered}
W_{\mathrm{suv}}^{(2 \mathrm{n})}\left(E^{*}\right) \sim \frac{\Gamma_{\mathrm{n}}^{(1 \mathrm{n})}\left(E^{*}\right)}{\Gamma_{\mathrm{Tot}}^{(1 \mathrm{n})}\left(E^{*}\right)}\left\langle\frac{\Gamma_{\mathrm{n}}^{(2 \mathrm{n})}\left(E^{*}-B_{\mathrm{n}}-\epsilon\right)}{\Gamma_{\mathrm{Tot}}^{(2 \mathrm{n})}\left(E^{*}-B_{\mathrm{n}}-\epsilon\right)} W_{<}^{(3 \mathrm{rd})}\left(E^{*}-B_{\mathrm{n}}-\epsilon\right)\right\rangle_{\epsilon}, \\
\sim \frac{\Gamma_{\mathrm{n}}^{(1 \mathrm{n})}\left(E^{*}\right)}{\Gamma_{\mathrm{Tot}}^{(1 \mathrm{n})}\left(E^{*}\right)} \frac{\Gamma_{\mathrm{n}}^{(2 \mathrm{n})}\left(E^{*}-B_{\mathrm{n}}-<\epsilon>\right)}{\Gamma_{\mathrm{Tot}}^{(2 \mathrm{n})}\left(E^{*}-B_{\mathrm{n}}-<\epsilon>\right)} W_{<}^{(3 \mathrm{rd})}\left(E^{*}-B_{\mathrm{n}}-<\epsilon>\right),
\end{gathered}
$$

where $W_{<}^{(3 r d)}$ is the probability for the third-chance fission or neutron emission, such as that given in eqs. 8 and $9,<\cdots>$ is the ensemble average, and $\epsilon$ is the kinetic energy of the second emitted neutron. The quantity $\langle\epsilon>$ can be obtained as $<\epsilon>=2 T$, assuming the Boltzman distribution for the neutron evaporation spectrum.

\subsection{Survival probability for radiative-capture reaction}

In an analogy with the calculation of the $1 \mathrm{n}$ reaction, we calculate the survival probability $W_{\text {suv }}^{(0 \mathrm{n})}$ for the radiativecapture reaction as the product of the probability of gamma emission and survival probability against the second-chance 


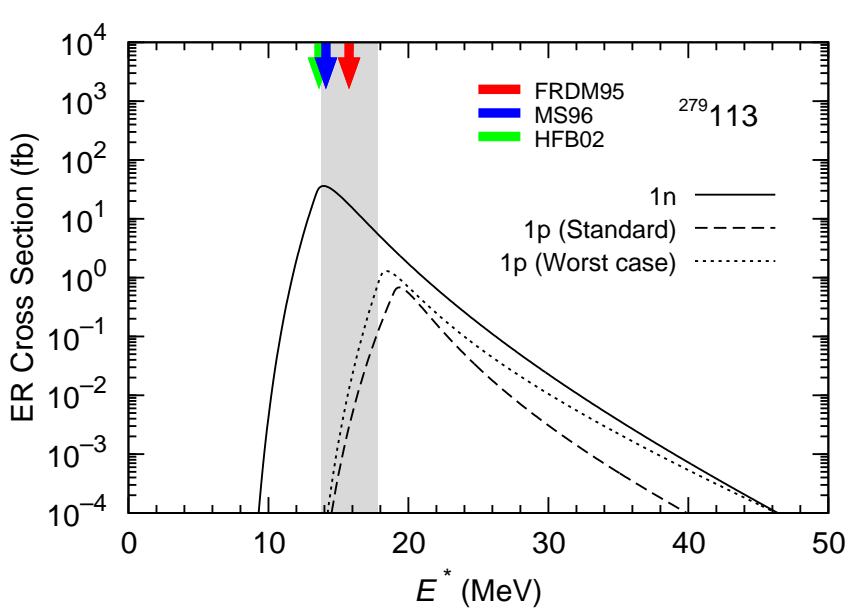

Fig. 2. (Color online) Evaporation residue cross sections for one-proton and one-neutron emissions versus excitation energy for the compound nucleus ${ }^{279} 113$. The solid and dashed lines respectively indicate the calculated results for the $1 \mathrm{n}$ and $1 \mathrm{p}$ reaction channels with the standard leveldensity parameter and fission barrier height. The dotted line shows the calculated result of the $1 \mathrm{p}$ reaction channel for the worst-case scenario with $a_{\mathrm{p}} \times 1.1$. The dark-gray, black, and gray arrows indicate the excitation energies corresponding to the experimental incident energies calculated using FRDM95, MS06, and HFB02, respectively. The light-gray area denotes the incident-energy distribution for the worst-case scenario.

fission or neutron emission after a gamma-ray emission, $W_{<}^{(0 n)}$, given by

$$
W_{\mathrm{suv}}^{(0 \mathrm{n})}\left(E^{*}\right)=\frac{\Gamma_{\gamma}\left(E^{*}\right)}{\Gamma_{\mathrm{Tot}}\left(E^{*}\right)} W_{<}^{(0 \mathrm{n})}\left(E^{*}\right) .
$$

We can calculate $W_{<}^{(0 n)}$ with the probability below the fission or neutron emission threshold energy after a gamma-ray emission using the gamma-ray spectrum of eq. $5 . W_{<}^{(0 n)}$ is thus given by

$$
W_{<}^{(0 \mathrm{n})}=\frac{\int_{E_{\mathrm{th}}}^{U^{*}} w_{\gamma}\left(U^{*}, \epsilon_{\gamma}\right) d \epsilon_{\gamma}}{\int_{0}^{U^{*}} w_{\gamma}\left(U^{*}, \epsilon_{\gamma}\right) d \epsilon_{\gamma}},
$$

where $E_{\mathrm{th}}$ is the threshold energy for the second-chance fission or neutron evaporation, whichever is lower.

\subsection{Expected value relative to In reaction channel}

To discuss the possibility of the non- 1 n reaction channel under actual experimental conditions, we calculate the expected value relative to the $1 \mathrm{n}$ reaction channel, taking into account the effect of the target thickness, given by

$$
P=\frac{\int_{E_{\min }^{*}}^{E_{\max }^{*}} \sigma_{\mathrm{ER}}\left(E^{*}\right) d E^{*}}{\int_{E_{\min }^{*}}^{E_{\max }^{*}} \sigma_{\mathrm{ER}}^{(1 \mathrm{n})}\left(E^{*}\right) d E^{*}},
$$

where $E_{\max }^{*}$ and $E_{\min }^{*}$ are the upper and lower bounds of the incident-energy distribution due to the target thickness, respectively. One advantage of this equation is that we can partially reduce the effects of uncertainties in $\sigma_{\text {cap }}$ and $P_{\text {diff }}$ defined in eq. 1.

\section{Result and Discussion}

\subsection{Probability for one-proton evaporation channel}

To discuss the probability for the $1 p$ channel relative to the 1 n channel, we calculate $\sigma_{\mathrm{ER}}^{(1 \mathrm{p})}$ and compare it with $\sigma_{\mathrm{ER}}^{(1 \mathrm{n})}$.

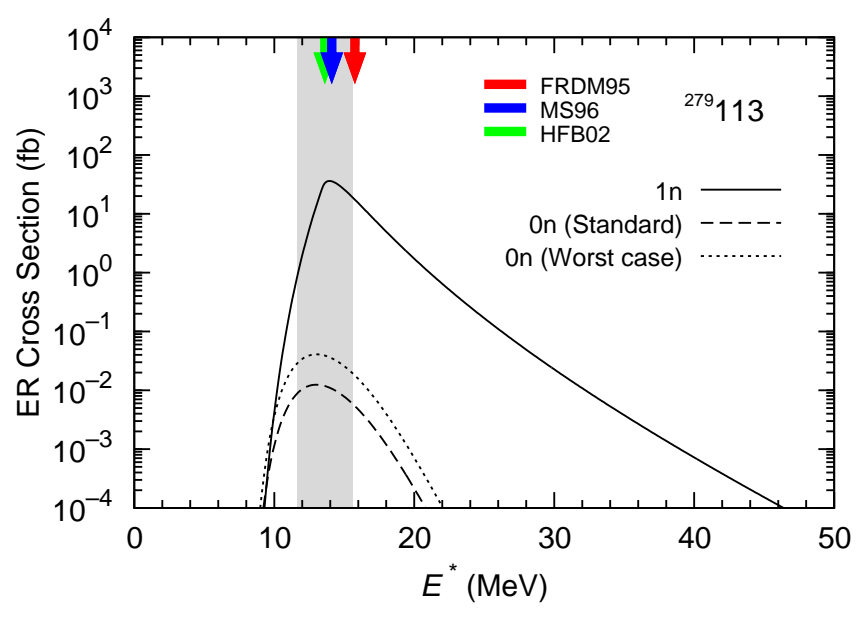

Fig. 3. (Color online) Evaporation residue cross sections for the radiativecapture and one-neutron emission channels versus excitation energies of the compound nucleus ${ }^{279} 113$. The solid and dashed lines respectively indicate the calculated results for the $1 \mathrm{n}$ and 0 n reaction channels with standard parameters. The dotted line shows the results with $a_{\gamma} \times 1.1$ and a decrease in fission barrier height. The arrows and the gray area are the same as those in Fig. 1.

Figure 2 shows the calculated result versus excitation energy for the nucleus ${ }^{279} 113$. The solid and dashed lines indicate the results for the $1 \mathrm{n}$ and $1 \mathrm{p}$ reaction channels with the standard level-density parameter and fission barrier height, respectively. For the standard parameter set, the excitation energy of the worst-case scenario is estimated to be $17.76 \mathrm{MeV}$ with FRDM95, and the resultant $E_{\max }^{*}$ and $E_{\min }^{*}$ are 19.76 and 15.76 MeV, respectively. The incident-energy distribution is denoted by the light-gray area in the figure. In this case, we determine the expected value of the $1 \mathrm{p}$ reaction channel relative to the $1 \mathrm{n}$ reaction channel, $P^{(1 \mathrm{p} / 1 \mathrm{n})}$, to be $8.51 \times 10^{-4}$, which is a negligibly small probability for one-proton evaporation.

To determine the uncertainty originating from the leveldensity parameter and fission barrier height, we calculate $\sigma_{\mathrm{ER}}^{(1 \mathrm{p})}$ with $a_{\mathrm{p}} \times 1.1$ and a decrease in fission barrier height of ${ }^{278} 112$ by $1 \mathrm{MeV}$ (We also change consistently the shell correction energy at the ground state). The calculated results are indicated by the dotted line in Fig. 2. Even in this case, the expected value of one-proton emission relative to one-neutron emission is less than $4.97 \times 10^{-3}$. The branching ratio of oneproton emission is thus negligibly small.

\subsection{Probability for radiative-capture channel}

Here, we discuss the probability of the radiative-capture and $1 \mathrm{n}$ reaction channels. We calculate $\sigma_{\mathrm{ER}}^{(\mathrm{n})}$ and compare it with $\sigma_{\mathrm{ER}}^{(0 \mathrm{n})}$. Figure 3 shows the calculated result. The solid and dashed lines indicate the calculated results for the $1 \mathrm{n}$ and $0 \mathrm{n}$ reaction channels with the standard level-density parameter and the fission barrier height, respectively. The excitation energy in the worst-case scenario is estimated to be $13.61 \mathrm{MeV}$ with HFB02, and the resultant $E_{\max }$ and $E_{\min }$ are 15.61 and $11.61 \mathrm{MeV}$, respectively. For this case, the expected value of the 0 reaction channel relative to the 1 n reaction channel, $P^{(0 \mathrm{n} / 1 \mathrm{n})}$, is $5.14 \times 10^{-4}$.

We also increase the level-density parameter by $10 \%$ to estimate the worst-case scenario. For the radiative-capture re- 


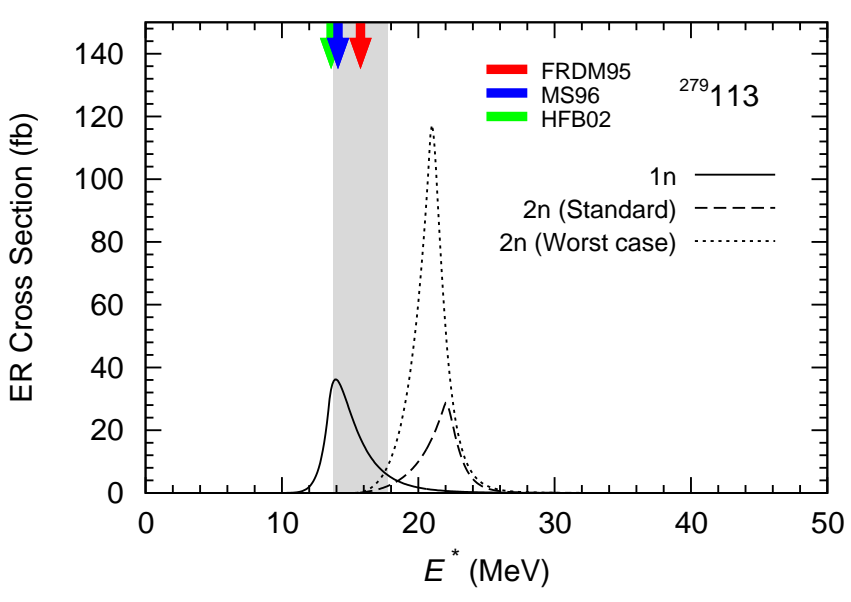

Fig. 4. (Color online) Evaporation residue cross sections for the $2 \mathrm{n}$ and $1 \mathrm{n}$ reactions versus excitation energy of the compound nucleus ${ }^{279} 113$. The solid and dashed lines respectively indicate the calculated results for the $1 \mathrm{n}$ and $2 \mathrm{n}$ reaction channels with standard parameters. The dashed line shows the result obtained with the level-density parameter $a_{n} \times 1.1$ for the $2 \mathrm{n}$ reaction and a decrease in fission barrier height by $1 \mathrm{MeV}$ (We also change the corresponding shell correction energy at the ground state). The arrows and the light-gray area are the same as those in Fig. 1.

action, we do not change the height of the fission barrier of ${ }^{279} 113$ for the calculation of the second-chance fission, because such changes affect the calculated result for the 1n reaction channel. The calculated result is indicated by the dotted line in the figure. For this worst-case scenario, $P^{(0 \mathrm{n} / 1 \mathrm{n})}$ is less than $1.70 \times 10^{-3}$, which is so small that the radiative-capture channel can be excluded.

\subsection{Probability for two-neutron evaporation channel}

To study the probability for the two-neutron evaporation channel, we calculate $\sigma_{\mathrm{ER}}^{(2 \mathrm{n})}$. To estimate the level-density parameter at the saddle point, we use the elongation of the major semi axis at the saddle configuration relative to the spherical shape, which is $2.5 \mathrm{fm}$, the recommended value for the deformed nucleus at the ground state in ref. 7. We also determine the sensitivity of the calculation to this parameter and observe that it is negligibly small.

We first calculate $\sigma_{\mathrm{ER}}^{(2 \mathrm{n})}$ with the standard parameter set and show the results in Fig. 4. The solid and dashed lines respectively indicate the calculated results for the $1 \mathrm{n}$ and $2 \mathrm{n}$ reaction channels with standard parameters. In the figure, we see that the probability of the $2 \mathrm{n}$ reaction cannot be excluded only for the excitation energy estimated with FRDM95. The other two estimations are well below the threshold energy for the $2 \mathrm{n}$ reaction, even if we take into account the effect of the target thickness. The expected value of the $2 \mathrm{n}$ reaction channel relative to the $1 \mathrm{n}$ reaction channel, $P^{(2 \mathrm{n} / 1 \mathrm{n})}$, is $2.44 \times 10^{-2}$; thus, we can safely neglect the possibility of the $2 \mathrm{n}$ reaction channel.

We next discuss the uncertainty originating from the leveldensity parameter and the fission barrier height. We increase $a_{\mathrm{n}}$ for the $2 \mathrm{n}$ reaction channel by $10 \%$ and decrease the height of the fission barrier of ${ }^{277} 113$ and the shell correction energy by $1 \mathrm{MeV}$. The calculated result is indicated by the dotted line in Fig. 4. In the figure, we can see an unrealistic enhancement of the $2 \mathrm{n}$ reaction channel, compared with the fact that in all the experiments of the cold fusion reactions, the observed peak values of the ER cross section for the $1 \mathrm{n}$ reaction are higher than or comparable to that of the 2 n reaction. ${ }^{1)}$ For this case, $P^{(2 \mathrm{n} / \mathrm{ln})}=7.91 \times 10^{-2}$, which is still a fairly small probability for the $2 \mathrm{n}$ reaction despite such unrealistic parameter values.

\subsection{Probability for total non-1n evaporation channel}

Finally, we calculate the total non-1n branching ratio, $P^{(\text {non-1n/1n) }}$, using the sum of the probabilities for the proton, radiative-capture, and $2 \mathrm{n}$ reaction channels. For the standard parameter set, we obtain $P^{(\text {non-1n/1n) }}=2.55 \times 10^{-2}$, indicating that the dominant component of the non- $1 \mathrm{n}$ channels is the $2 \mathrm{n}$ evaporation channel.

For the worst-case scenario, we simultaneously use all the parameter sets for each channel discussed in the previous sections. Although we independently change the model parameters for each channel in discussing the worst-case scenario, their effects on the other channels are very small, because we only change the parameters for the different residual compound nuclei at the final state and fix those for the 1n evaporation channel in order to reproduce the experimental data. With all the parameters for the worst-case scenario, we calculate the expected values at the excitation energies estimated with FRDM95 and obtain $P^{(1 \mathrm{p} / 1 \mathrm{n})}=4.97 \times 10^{-3}$, $P^{(0 \mathrm{n} / 1 \mathrm{n})}=9.94 \times 10^{-4}$, and $P^{(2 \mathrm{n} / 1 \mathrm{n})}=7.92 \times 10^{-2}$. The total non- $1 \mathrm{n}$ branching ratio is then $P^{(\mathrm{non}-1 \mathrm{n} / 1 \mathrm{n})}=8.51 \times 10^{-2}$, which is still a fairly small probability.

\section{Conclusions}

We have discussed the possibilities of proton emission, radiative-capture, and two-neutron emission channels in the de-excitation of the compound nucleus ${ }^{279} 113$ produced in the ${ }^{209} \mathrm{Bi}+{ }^{70} \mathrm{Zn}$ reaction reported in ref. 3. To this end, we extend the FBD model to those channels. We vary the ratio of the level-density parameter to the height of the fission barrier to determine its effect on calculations and determine the upper bounds of those channels.

To discuss the branching ratio, we calculate the expected value of the other reaction channel relative to the $1 \mathrm{n}$ reaction channel under actual experimental conditions. For the oneproton emission channel, we obtain $P^{(1 \mathrm{p} / 1 \mathrm{n})}=8.51 \times 10^{-4}$ and at most $4.97 \times 10^{-3}$, indicating that the probability of the oneproton emission channel can be ignored. For the radiativecapture channel, we obtain $P^{(0 \mathrm{n} / \mathrm{ln})}=5.14 \times 10^{-4}$ and at most $1.70 \times 10^{-3}$. We thus consider that the possibility of the radiative-capture channel can be excluded. For the twoneutron emission channel, we obtain $P^{(2 n / 1 n)}=2.44 \times 10^{-2}$ and at most $7.91 \times 10^{-2}$, which is a fairly small probability despite the unrealistic parameter values. For the total non-1n branching ratio, we obtain $P^{(\text {non-1n/1n) }}=2.55 \times 10^{-2}$ and at most $8.51 \times 10^{-2}$. We conclude that the $2 \mathrm{n}$ reaction is the main branch in the non- $1 \mathrm{n}$ reaction channels.

For the estimations of the worst-case scenario, the most sensitive parameter is the nuclear mass. The development of a mass model would largely improve the determination of the optimum bombarding energy in synthesizing new elements.

\section{Acknowledgments}

We dedicated this paper to the memory of Władek Świątecki, whose works on fission and superheavy elements were an inspiration to us throughout our careers. We would like to thank P. Möller for valuable discussions. 
1) S. Hofmann and G. Mun̈zenberg: Rev. Mod. Phys. 72 (2000) 733.

2) Y. Oganessian: J. Phys. G: Nucl. Part. Phys. 24 (2007) R165.

3) K. Morita, K. Morimoto, D. Kaji, T. Akiyama, S. Goto, H. Haba, E. Ideguchi, R. Kanungo, K. Katori, H. Koura, H. Kudo, T. Ohnishi, A. Ozawa, T. Suda, K. Sueki, H. Xu, T. Yamaguchi, A. Yoneda, A. Yoshida and Y. Zhao: J. Phys. Soc. Jpn. 73 (2004) 2593.

4) K. Morita, K. Morimoto, D. Kaji, T. Akiyama, S. Goto, H. Haba, E. Ideguchi, K. Katori, H. Koura, H. Kikunaga, H. Kudo, T. Ohnishi, A. Ozawa, N. Sato, T. Suda, K. Sueki, F. Tokanai, T. Yamaguchi, A. Yoneda, and A. Yoshida: J. Phys. Soc. Jpn. 76 (2007) 045001.

5) K. Morita, K. Morimoto, D. Kaji, H. Haba, K. Oseki, Y. Kudou, N. Sato, T. Sumita, A. Yoneda, T. Ichikawa, Y. Fujimori, S. Goto, E. Ideguchi, Y. Kasamatsu, K. Katori, Y. Komori, H. Koura, H. Kudo, K. Ooe, A. Ozawa, F. Tokanai, K. Tsukada, T. Yamaguchi, and A. Yoshida: J. Phys. Soc. Jpn. 78 (2009) 064201.

6) W. J. Świątecki, K. Siwek-Wilczyńska, and J. Wilczyński: Acta Phys. Pol. 34 (2003) 2049.

7) W. J. Świa̧tecki, K. Siwek-Wilczyńska, and J. Wilczyński: Phys. Rev. C 71 (2005) 014602.

8) S. L. Nelson, K. E. Gregorich, I. Dragojevic, M. A. Garcia, J. M. Gates, R. Sudowe, and H. Nitsche: Phys. Rev. Lett. 100 (2008) 022501.

9) I. Dragojevic, K. E. Gregorich, Ch. E. Dullmann, M. A. Garcia, J. M. Gates, S. L. Nelson, L. Stavsetra, R. Sudowe, and H. Nitsche: Phys. Rev. C 78 (2008) 024605.

10) S. L. Nelson, C. M. Folden III, K. E. Gregorich, I. Dragojevic, Ch. E. Dullmann, R. Eichler, M. A. Garcia, J. M. Gates, R. Sudowe, and H. Nitsche: Phys. Rev. C 78 (2008) 024606.

11) J. M. Gates, S. L. Nelson, K. E. Gregorich, I. Dragojevic, Ch. E. Dullmann, P. A. Ellison, C. M. Folden III, M. A. Garcia, L. Stavsetra, R. Sudowe, D. C. Hoffman, and H. Nitsche: Phys. Rev. C 78 (2008) 034604.

12) I. Dragojevic, K. E. Gregorich, Ch. E. Dullmann, J. Dvorak, P. A. Ellison, J. M. Gates, S. L. Nelson, L. Stavsetra, and H. Nitsche: Phys. Rev. C 79 (2009) 011602.

13) C. M. Folden III, I. Dragojevic, Ch. E. Dullmann, R. Eichler, M. A. Garcia, J. M. Gates, S. L. Nelson, R. Sudowe, K. E. Gregorich, D. C. Hoffman, and H. Nitsche: Phys. Rev. C 79 (2009) 027602 (2009)

14) S. L. Nelson, K. E. Gregorich, I. Dragojevic, J. Dvorak, P. A. Ellison, M. A. Garcia, J. M. Gates, L. Stavsetra, M. N. Ali, and H. Nitsche: Phys. Rev. C 79 (2009) 027605.

15) W. J. Świątecki, K. Siwek-Wilczyńska, and J. Wilczyński: Phys. Rev. C 78 (2008) 054604.

16) G. G. Adamian and N. V. Antonenko: Phys. Rev. C 81 (2010) 019803.
17) W. J. Świątecki, K. Siwek-Wilczyńska, and J. Wilczyński: Phys. Rev. C 81 (2010) 019804.

18) R. Vandenbosch and J. R. Huizenga: Nuclear fission (Academic Press, Inc., London, 1973).

19) P. Möller, J. R. Nix, W. D. Myers, and W. J. Świątecki: At. Data Nucl. Data Tables 59 (1995) 185.

20) S. Goriely, M. Samyn, P. -H. Heenen, J. M. Pearson, and F. Tondeur: Phys. Rev. C 66 (2002) 024326.

21) Handbook for Calculations of Nuclear Reaction Data RIPL-2, IAEA-TECDOC-1506, IAEA, Bienna (2006): see also. http://wwwnds.iaea.org/RIPL-2/.

22) W. D. Myers and W. J. Świą̧ecki: Nucl. Phys. A601 (1996) 141; Lawrence Berkely Laboratory preprint LBL-36803, December 1994 (unpublished), available on http:/ie.lbl.gov/txt/ms.txt.

23) P. Möller, A. J. Sierk, T. Ichikawa, A. Iwamoto, R. Bengtsson, H. Uhrenholt, and S. Åberg: Phys. Rev. C 79 (2009) 064304.

24) P. Möller, A. J. Sierk, and A. Iwamoto: Phys. Rev. Lett. 92 (2004) 072501.

25) K. Morita, K. Morimoto, D. Kaji, H. Haba, E. Ideguchi, R. Kanungo, K. Katori, H. Koura,, H. Kudo, T. Ohnishi, A. Ozawa, T. Suda, K. Sueki, I. Tanihata, H. Xu, A.V. Yeremin, A. Yoneda, A. Yoshida, Y.-L. Zhao and T. Zheng: Eur. Phys. J. A 21 (2004) 257.

26) K. Morita, K. Morimoto, D. Kaji1, H. Haba, E. Ideguchi, J. C. Peter, R. Kanungo, K. Katori, H. Koura, H. Kudo, T. Ohnishi, A. Ozawa, T. Suda, K. Sueki, I. Tanihata, H. Xu, A. V. Yeremin, A. Yoneda, A. Yoshida, Y.L. Zhao, T. Zheng, S. Goto, and F. Tokanai: J. Phys. Soc. Jpn. 73 (2004) 1738 .

27) K. Morita, K. Morimoto, D. Kaji, T. Akiyama, S. Goto, H. Haba, E. Ideguchi, K. Katori, H. Koura, H. Kudo, T. Ohnishi, A. Ozawa, T. Suda, K. Sueki, F. Tokanai, T. Yamaguchi, A. Yoneda, and A. Yoshida: J. Phys. Soc. Jpn. 76 (2007) 043201.

28) K. Morita: private communication.

29) G. Audia, A. H. Wapstrab, and C. Thibaulta: Nucl. Phys. A729 (2003) 337.

30) L. C. Vaz and J. M. Alexander: Z. Phys. A 318 (1984) 318.

31) L. G. Moretto: Phys. Lett. 40B (1972) 185: Nucl. Phys. A247 (1975) 211.

32) W. E. Parker, M. Kaplan, D. J. Moses, G. L. Rana, D. Logan, R. Lacey, J. M. Alexander, D. M. de Castro Rizzo, P. DeYoung, R. J. Welberry, and J. T. Boger: Phys. Rev. C 44 (1991) 774.

33) K. -H. Schmidt and W. Morawek: Rep. Prog. Phys. 54 (1991) 949.

34) W. D. Myers: Droplet Model of Atomic Nuclei (IFI/Plenum, New York, 1977). 